\title{
Pregnancy-related anxiety during COVID-19: A nationwide survey of 2,740 pregnant women
}

Cheryl A Moyer ( $\square$ camoyer@umich.edu )

University of Michigan

\section{Sarah D Compton}

University of Michigan

Elizabeth Kaselitz

University of Michigan

Maria Muzik

University of Michigan

\section{Research Article}

Keywords: Perinatal anxiety, Perinatal mental health, Maternal mental health, Pregnancy

Posted Date: June 26th, 2020

DOl: https://doi.org/10.21203/rs.3.rs-37887/v1

License: (c) (i) This work is licensed under a Creative Commons Attribution 4.0 International License. Read Full License 


\section{Abstract}

Purpose: To explore the impact of the COVID-19 pandemic on pregnant women's anxiety and identify factors most strongly associated with greater changes in anxiety.

Methods: An anonymous, online, survey of pregnant women (distributed April 3 - 24, 2020) included a modified Pregnancy-Related Anxiety Scale (PRAS) reflecting respondents' perception of pregnancy anxiety before COVID-19 and a current assessment of pregnancy-related anxiety. The difference between these scores was used as the outcome variable. Data were analysed using bivariate and multivariate linear regression analyses.

Results: 2,740 pregnant women from 47 states completed the survey. 25.8\% ( $\mathrm{N}=706)$ stopped in-person visits, $15.2 \%$ used video visits $(\mathrm{N}=415)$, and $31.8 \%(\mathrm{~N}=817)$ used phone visits for prenatal care as a result of COVID-19. Those planning a hospital birth dropped from 2641 (96.4\%) to 2400 (87.7\%) following COVID-19. More than half of women reported increased stress about food running out $(59.2 \%, \mathrm{~N}=1622)$, losing a job or household income $(63.7 \%, \mathrm{~N}=1745)$, or loss of childcare $(56.3 \%, \mathrm{~N}=1543)$. More than a third reported increasing stress about conflict between household members $(37.5 \%, \mathrm{~N}=1028)$, and $93 \%$ $(\mathrm{N}=2556)$ reported increased stress about getting infected with COVID-19. Slightly less than half of respondents (either selves or family members) were healthcare workers $(41.4 \%, \mathrm{~N}=1133)$ or worked in essential services (45.5\%, $\mathrm{N}=1246)$. In multivariate analysis, those reporting higher agreement with COVID-19-related stressors had greater changes in pre- to post-COVID-19 pregnancy-related anxiety.

Conclusions: The COVID-19 pandemic is profoundly affecting pregnant women's mental health, and factors independent of pregnancy appear to be driving changes in pregnancy-specific anxiety.

\section{Introduction}

Researchers have been racing to understand the impact of COVID-19 disease on pregnant women and fetuses. Early publications focus on understanding incidence and severity during pregnancy, based on limited COVID-19 data and data from other coronaviruses.(Rasmussen et al. 2020) While current research suggests that pregnant women are not at an elevated risk for severe disease,(Schwartz 2020) in New York City, the majority of pregnant women who tested positive for COVID were asymptomatic. (Breslin et al. 2020) Thus hospital systems are dealing with the potential of asymptomatic pregnant women spreading COVID-19 to healthcare providers, other women, and support people during labor. Some institutions are conducting universal testing(Sutton et al, 2020) or have instituted strict policies regarding labor companions. Two New York hospitals banned all birthing partners for a time,(Caron et al. 2020) and after some of those restrictions were lifted, still banned visitors in the postpartum unit.(NYP 2020) Such a rapidly-changing context may lead to increased anxiety among pregnant women about their labor and delivery, a time of already increased stress.

The impact of the COVID-19 pandemic on pregnancy-related anxiety has yet to be systematically studied. Saccone et al. (2020) published a small study of 100 pregnant women in Italy, in which more 
than half rated the psychological impact of COVID-19 as severe, and two-thirds were more anxious than normal.(Saccone et al. 2020) However, the study design precluded a deeper exploration of what aspects of the COVID-19 outbreak were driving increased anxiety. While another study in China explored the drivers of COVID-19-related anxiety(Wang et al. 2020), pregnant women were not included in this sample.

This research was designed to explore the impact of the COVID-19 pandemic on pregnant women's anxiety, as well as to identify the factors that were most strongly associated with a greater increase in anxiety.

\section{Materials And Methods}

Study design: Cross-sectional, anonymous, online survey of pregnant women identified via FaceBook, Twitter, and pregnancy-related online peer and professional communities April 3 - 24, 2020.

Setting: English-language online survey open to any pregnant woman, regardless of geographic location.

Participants: Self-identified pregnant women able to complete an online survey in English, recruited via FaceBook and Twitter, including pregnancy-specific FaceBook groups and snowball sampling among pregnant respondents. The survey link was also distributed to pregnancy-specific professional communities for distribution. We sought additional gatekeepers in rural, urban, minority, and low-income populations, asking them to circulate the survey link within their networks.

\section{Data sources / measurement:}

The survey was developed using an iterative process with expert review, including previously used demographic and health questions and a modification of the Pregnancy-Related Anxiety Scale (PRAS) (Rini et al. 1999). The survey was formatted for use in Qualtrics@ and assessed demographic and pregnancy-related factors, anxiety, and psychosocial risk factors.

Demographic and pregnancy-related measures: The survey included basic demographic questions, pregnancy-related background questions, and questions regarding the impact of COVID-19 on utilization of prenatal care and intentions for delivery location. (See Table 1.)

Anxiety Measures: Anxiety was measured in several ways. First, we used a 10-point Visual Analog Scale (VAS) to assess anxiety about being pregnant during the COVID-19 pandemic(Williams et al. 2010), with 1 being not at all anxious and 10 being extremely anxious. We used another 10-point VAS to assess anxiety about giving birth during the COVID-19 pandemic. We also used a modified version of the PRAS(Rini et al. 1999), asking women to think back before COVID-19 and then repeating the questions given the current climate. (See Appendix 1.) 
Psychosocial Risk Factor Measures: Five questions assessed psychosocial factors that may contribute to COVID-specific anxiety, including concerns about 1) food running out or being unavailable; 2) losing a job or decrease in family income; 3 ) loss of childcare / taking care of children at home; 4) tension / conflict between household members; and 5) self, baby, or family getting infected with COVID-19. Each was assessed via Likert scale, from strongly disagree (1) to strongly agree (5). A final question assessed whether the respondent or a family member works in healthcare with potential exposure to COVID-19 patients, whether the respondent or a family member works in essential services that require leaving home, whether the respondent lives in a state with 'shelter in place' orders, whether the respondent has been practicing social distancing, and whether she lives in a community or city with a high number of COVID-19 cases.

Outcome variable: We utilized the net change in PRAS score (current COVID-pre-COVID) as our outcome variable. Both the pre-COVID and current PRAS scores were calculated by reverse scoring the 4 positively worded items within the scale and then summing the 8 items, each with a range of 1-5 points. This yielded a possible range of 8-40 points, with higher scores indicating a higher level of anxiety. The preCOVID PRAS score was then subtracted from the current COVID PRAS score, creating a normally distributed range of values from -9 to 17 .

Predictor variables: Tables 1 and 2 illustrate the predictor variables. "Previous health conditions" reflected any of the following before pregnancy: high blood pressure, heart disease, asthma, diabetes, cancer, autoimmune diseases, or HIV/AIDS. "Previous depression / anxiety" was determined by the respondent indicating 'yes' to the following question: "Before this pregnancy, had you been treated for depression or anxiety or other mental health issues?" The variable "pregnancy health conditions" was indicative of the respondent answering 'yes' to any of the following: "Since becoming pregnant, have you been diagnosed with any of the following conditions? High blood pressure, preeclampsia, gestational diabetes, anemia, placenta previa, other diagnoses." "Pregnancy-diagnosed depression/anxiety" was indicated if the respondent answered 'yes' to the question, "Since becoming pregnant, have you been diagnosed with depression or anxiety?"

The variables "stopped in-person prenatal visits," "used video visits for prenatal care," and "used phone visits for prenatal care" were indicated by yes/no responses. The variables "food running out," "losing a job," "loss of childcare," "tension/conflict in house," and "getting infected" were determined from the percentage of respondents who either somewhat agreed or strongly agreed with statements about increased stress about each factor. Risk factors for COVID were indicated by affirmative responses to self or family member being a healthcare worker with potential exposure to COVID+ patients, self or family member working in essential services that requires leaving home, living in a state with 'shelter in place' orders, practicing social distancing, and living in a community/city with a high number of COVID-19 cases.

The variables "anxiety about pregnancy" and "anxiety about delivery" during COVID were determined using the 10-point VAS described previously. 
Study size: There was no a priori sample size determined.

Data Analysis / Statistical methods: All data were downloaded from Qualtrics and cleaned, removing records with more than $50 \%$ missing data. Basic descriptive statistics were calculated for all variables. As an internal validity check, the two anxiety questions assessed via visual analog scale were correlated with one another and with the difference in PRAS scores. Bivariate linear regression was conducted to compare how changes in PRAS scores were related to key variables of interest. Those variables found to be associated were used in multivariate linear regression to yield a final model. A p-value of 0.05 was taken for statistical significance.

Ethical Review and Reporting Criteria: All study materials and methods were reviewed and deemed exempt from ongoing review by the University of Michigan Institutional Review Board (HUM00179610). STROBE criteria were used in the planning, implementing, and reporting of this research. (von Elm 2008)

\section{Results}

A total of 4,274 women responded, 1114 who responded to less than half of the survey and 45 who indicated being less than 5 weeks pregnant. Of the remaining 3,115, 3,070 answered the question regarding country of residence: 330 resided outside the United States (U.S.) and 2,740 resided within the U.S. For the purpose of the present paper, analyses are restricted to respondents from within the U.S. $(n=$ 2740).

Table 1 displays the demographics and pregnancy-related characteristics of respondents. Mean age was $32.7,41.2 \%(\mathrm{~N}=1128)$ were in their third trimester, and $15.6 \%(\mathrm{~N}=429)$ were experiencing their first pregnancy. Respondents came from 47 states, most were white $(87.7 \%, \mathrm{~N}=2388)$ and half were college graduates or less $(49.5 \%, \mathrm{~N}=1358)$. More than a third had previously diagnosed depression, anxiety, or other mental health issue $(35.9 \%, \mathrm{~N}=982)$. Approximately a quarter $(25.8 \%, \mathrm{~N}=706)$ reported stopping in-person prenatal care visits since COVID, $15.2 \%$ had used a video visit $(\mathrm{N}=415)$, and $31.8 \%(\mathrm{~N}=817)$ had used a phone visit for prenatal care. Those planning to deliver in a hospital dropped from 2641 (96.4\%) prior to COVID to 2400 (87.7\%) following COVID.

Table 1 also illustrates the beta coefficients for each variable when regressed against the difference in pre-COVID and current PRAS scores. The factors significantly associated with greater changes in PRAS scores included being in the third trimester, having a history of or recent diagnosis of depresson or anxiety, having stopped in-person prenatal care, or having used the phone for prenatal care. Women who originally planned to give birth at a hospital and those who moved away from planning to deliver at a hospital had significantly greater changes in PRAS scores. Factors associated with smaller changes in PRAS scores included maternal age, higher education, being married, and planning to give birth at home.

Anxiety scores shown in Table 2 indicate that women are anxious about being pregnant during COVID (mean 6.5 on a scale of $1-10,95 \% \mathrm{Cl} 6.4,6.6$ ), but more anxious about giving birth during COVID (mean 7.6 on a scale of $1-10,95 \%$. Cl 7.5, 7.6). Mean pre-COVID PRAS scores were 20.6 (95\% Cl 20.5, 20.7), and 
mean current PRAS scores were 23.9 ( $95 \% \mathrm{Cl} 23.8,24.0)$. When comparing measures of anxiety against our outcome measure (difference in PRAS scores) as a consistency check, the relationships were as expected. Greater anxiety about being pregnant and giving birth were significantly associated with greater changes in PRAS scores $(p<0.001)$.

In terms of psychosocial risk factor indicators, more than half of respondents reported increased stress about food running out or not being available $(59.2 \%, N=1622)$, losing a job or loss of household income $(63.7 \%, N=1745)$, or loss of childcare $(56.3 \%, N=1543)$. (See Table 2.) More than a third reported increasing stress about conflict between household members $(37.5 \%, \mathrm{~N}=1028)$. Ninety three percent $(\mathrm{N}=$ 2556) reported increased stress about getting infected with COVID-19. When asked about risk factors for COVID-19, slightly less than half of respondents (either themselves or their family members) were healthcare workers $(41.4 \%, N=1133)$ or essential workers required to leave home $(45.5 \%, N=1246)$. Forty two percent $(\mathrm{N}=1149)$ reported living a community or city with a high number of COVID-19 cases, $77.3 \%(N=2117)$ reported living in a state with 'shelter in place' orders, and $87.4 \%(N=2395)$ reported practicing social distancing.

The variables significantly associated with greater changes in PRAS scores included increased stress about food availability, losing a job, loss of childcare, tension in the home, getting infected, self or family member being an essential worker, or living in a community with a high number of COVID-19 cases. (Table 2.) The variables associated with smaller changes in PRAS scores included living in a state with shelter in place orders or practicing social distancing.

Table 3 illustrates that even after controlling for age, education, being in the third trimester, and previous mental health diagnoses, those reporting higher agreement with COVID-related stressors are significantly more likely to have a greater change in pregnancy-related anxiety. Women who report increased stress about food availability, tension/conflict within the home, and potentially getting infected with COVID-19 had significantly greater changes in PRAS scores, and the interaction term is significant for women who have less than a high school education, suggesting these women are particularly impacted by anxiety about job or income loss. In addition, women who themselves or family members are essential workers or who report living in a high COVID-19 area had greater changes in pregnancy-related anxiety. Notably, women who changed their birth plan away from delivering in a hospital had a significantly greater change in their pregnancy-related anxiety scale score. At the same time, controlling for all other factors, practicing social distancing was significantly associated with smaller changes in pre- to current PRAS scores.

\section{Discussion}

This study found high levels of pregnancy-related anxiety among 2740 pregnant women from 47 states surveyed during the height of the COVID-19 epidemic in the U.S. The factors most strongly associated with greater changes in pregnancy-related anxiety included COVID-19-related issues, such as stopping face-to-face prenatal visits and changing birth plan away from delivering in a hospital, as well as fear of 
food running out, increased tension / conflict in the home, or fear of getting infected. In addition, self or family member being an essential worker or living in a location with a large number of COVID-19 cases were also significant drivers of greater changes in pregnancy-related anxiety scores. These factors were significant even after controlling for age, education, previous history of depression and anxiety, and other factors.

Similarly to Saccone et al (2020)'s findings in Italy(Saccone et al. 2020), these findings indicate that the COVID-19 pandemic is having a profound impact on pregnancy-related anxiety. Prior research has established pregnancy-related anxiety as distinct from general anxiety or depression(Bayrampour et al 2016) and is more detrimental to maternal and child outcomes(Blair et al 2011; Kramer et al 2009; Lobel et al 2008) Two important contributors to pregnancy-related anxiety have been demomstrated: real or anticipated threat to pregnancy or its outcomes, and low perceived control. Both of these factors is amplified by the COVID-19 panedmic. For example, Harpel (2008) reported that women who perceived their pregnancies as high risk showed more anxiety, yet felt assured and less anxious if they had access to ultrasound exams. Our findings are consistent with this literature: Women who experienced their pregnancies as potentially threatened by COVID showed greater changes in pregnancy-related anxiety due to the COVID-19 outbreak. Similarly, women who experienced a COVID-related disruption in their anticipated pregnancy and labor experience (i.e., loss in control) showed greater changes in pregnancyrelated anxiety. However, the direction of these latter relationships is not clear. It is possible that women with the greatest post-COVID anxiety initiated the termination of face-to-face prenatal care, for example. Alternatively, it is also possible that the providers asked women to shift away from face-to-face visits, and that this perceived "withdrawal of care" underlies women's increased anxiety. Future research is needed to tease out the direction of effects.

Concerns about risk of infection were important drivers of anxiety, manifesting through changes in where women planned to give birth, as illustrated by the drop from $96.4 \%$ of women planning a hospital birth prior to COVID to $87.7 \%$ planning a hospital birth during COVID. Interestingly, women who had planned a home birth prior to COVID and those who planned a home birth during COVID showed some of the smallest changes in pre-COVID to current PRAS scores. We hypothesize that this may be indicative of a greater locus of control among this group, as these women may have translated their concern about getting infected in a hospital setting to actionable control over the situation by planning labor in a perceived "safe" home environment.

For providers, it is important to recognize that pregnant women's anxiety about the COVID-19 pandemic is likely to translate to more generalized pregnancy-related anxiety. Providers and the system as a whole need to be prepared to offer additional supportive services to pregnant women, especially as standard care is being reshaped due to social distancing (e.g., stopping face-to-face prenatal visits, limiting support during labor etc.) Our findings indicated a relationship between greater changes in anxiety and a reduced preference for delivering in a hospital, a finding that is important for providers to recognize. Explicit discussions about birth plans, what providers and health systems can do to keep women and their babies 
safe, and the cost-benefit analysis of hospital-based versus home births will be extremely valuable in maximizing positive outcomes and minimizing maternal anxiety.

In addition, women with increased anxiety during pregnancy are known to be at increased risk for postpartum depression or other mood disorders(Grigoriadis et al 2019), suggesting ongoing monitoring for postpartum depression and other mood disturbances will be important.(Grigoriadis et al 2019; Muzik and Borovska 2010)

Our findings contribute to a line of research documenting the effects of disaster, trauma and life stressors during pregnancy on maternal mental health,(Huizink et al 2008; Laplante et al, 2008; Yehuda et al. 2005; Muzik et al 2016; Seng et al. 2010) confirming the detrimental impact of the COVID-19 pandemic and its psychosocial ramifications, including heightened interfamilial tension and sociodemographic hardship, on increasing pregnancy-related anxiety. They also suggest, paralleling prior work, that living through a particularly stressful time may have additional impacts on women beyond the normative pregnancy worries, and that women may need additional supports to recover and thrive, especially if they are living in communities with fewer resources. Future research is warranted exploring the long-term effects of the anxiety associated with COVID-19 on women who were pregnant during the pandemic.

This study is a nation-wide sample of nearly 3,000 pregnant women at the peak of the COVID-19 pandemic in the United States, and it assesses not only their COVID-related anxieties, but also their pregnancy-related anxiety. It is the first study to explore the relationship between these factors in a systematic way. Nonetheless, our online convenience sample introduces a bias toward better educated, more technology-savvy respondents. Minority populations are underrepresented in this sample, and are also being impacted by COVID-19 more profoundly, and thus conclusions must be drawn with such a limitation in mind. Nonetheless, we had representation from 47 states, including women of varying ages, education, parity, and risk status, and we believe this study captures important information about the impact of COVID-19 on maternal mental health.

\section{Conclusion}

Pregnancy can be stressful for women during normal times, yet the COVID-19 pandemic has amplified pregnancy-related anxiety among many women. Healthcare providers will need to offer increased and ongoing support to their pregnant patients, even as in-person visits are curtailed and social distancing policies reduce opportunities for meaningful patient-provider interactions.

\section{Declarations}

Funding: Not applicable

Conflicts of interest/competing interests: None 
Ethics approval: All study materials and methods were reviewed and deemed exempt from ongoing review by the University of Michigan Institutional Review Board (HUM00179610).

Consent to participate: Participants were reminded of the elements of informed consent, but consent to participate was not documented due to the anonymous nature of the online survey

Consent for publication: Participants were assured that data would be anonymous and thus no identifying information would be used in any publication

Availability of data and materials: Data are available upon reasonable request

Code availability: Not applicable

Authors contributions:

CAM: Conceptualization, Methodology, Software, Formal Analysis, Writing-Original Draft, WritingReview and Editing

SDC: Conceptualization, Methodology, Formal Analysis, Writing-Original Draft, Writing-Review and Editing

EK: Conceptualization, Methodology, Writing-Original Draft, Writing-Review and Editing MM: Conceptualization, Methodology, Writing-Original Draft, Writing-Review and Editing

\section{References}

1. Bayrampour H, Ali E, McNeil DA, Benzies K, MacQueen G, Tough S (2016) Pregnancy-related anxiety: A concept analysis. Int J Nurs Stud 55:115-

2. Blair MM, Glynn LM, Sandman CA, Davis EP (2011) Prenatal maternal anxiety and early childhood temperament. Stress 14(6), 644-651

3. Breslin N, Baptiste C, Gyamfi-Bannerman C, Miller R, Martinez R, Bernstein K, et al. (2020) COVID-19 infection among asymptomatic and symptomatic pregnant women: Two weeks of confirmed presentations to an affiliated pair of New York City hospitals. Am J Obstet Gynecol MFM. 100118

4. Caron C, Van Syckle KV (2020) Some Pregnant Women in New York City Will Have to Deliver Babies Alone 2020 [Available from: https://www.nytimes.com/2020/03/24/parenting/coronavirus-laborbirth.html]

5. Grigoriadis S, Graves L, Peer M, et al. (2019) A systematic review and meta-analysis of the effects of antenatal anxiety on postpartum outcomes. Arch Womens Ment Health 22(5):543- 
6. Harpel TS (2008) Fear of the unknown: ultrasound and anxiety about fetal health. Health 3: 295312.

7. Huizink AC, Bartels M, Rose RJ, Pulkkinen L, Eriksson CJ, Kaprio J (2008) Chernobyl exposure as stressor during pregnancy and hormone levels in adolescent offspring. J Epid Comm Health 62(4):e5.

8. Kramer MS, Lydon J, Seguin L, Goulet L, Kahn SR, McNamara H, et al. (2009) Stress pathways to spontaneous preterm birth: the role of stressors, psychological distress, and stress hormones. Am J Epidemiol 169(11):1319-1326

9. Laplante DP, Brunet A, Schmitz N, Ciampi A, King S (2008) Project Ice Storm: prenatal maternal stress affects cognitive and linguistic functioning in 5 1/2-year-old children. $J$ Am Acad Child Adolesc Psychiatry 47(9):1063-1072

10. Lobel M, Cannella DL, Graham JE, DeVincent C, Schneider J, Meyer BA (2008) Pregnancy-specific stress, prenatal health behaviors, and birth outcomes. Health Psychol 27(5):604-615

11. Muzik M, Borovska S (2010) Perinatal depression: implications for child mental health. Ment Health Fam Med 7(4):239-247

12. Muzik M, McGinnis EW, Bocknek E, et al. (2016) PTSD symptoms across pregnancy and early postpartum among women with lifetime ptsd diagnosis. Depress Anxiety 33(7):584-591

13. New York Presbyterian. (2020) General Visitation Guidelines [updated April 27, 2020. Available from: https://www.nyp.org/coronavirus-information/coronavirus-visitor-policy-change

14. Oh W, Muzik M, McGinnis EW, Hamilton L, Menke RA, Rosenblum KL (2016) Comorbid trajectories of postpartum depression and PTSD among mothers with childhood trauma history: Course, predictors, processes and child adjustment. J Affect Disord 200:133-141

15. Rasmussen SA, Smulian JC, Lednicky JA, Wen TS, Jamieson DJ (2020) Coronavirus Disease 2019 (COVID-19) and pregnancy: what obstetricians need to know. Am J Obstet Gynecol

16. Rini CK, Dunkel-Schetter C, Wadhwa PD, Sandman CA (1999) Psychological adaptation and birth outcomes: the role of personal resrouces, stress, and sociocultural context in pregnancy. Health Psychology 18: 333-345

17. Saccone G, Florio A, Aiello F, Venturella R, De Angelis MC, Locci M, et al. (2020) Psychological Impact of COVID-19 in pregnant women. Am J Obstet Gynecol

18. Schwartz DA (2020) An Analysis of 38 Pregnant Women with COVID-19, Their Newborn Infants, and Maternal-Fetal Transmission of SARS-CoV-2: Maternal Coronavirus Infections and Pregnancy Outcomes. Arch Pathol Lab Med 
19. Seng JS, Rauch SA, Resnick H, et al. (2010) Exploring posttraumatic stress disorder symptom profile among pregnant women. J Psychosom Obstet Gynaecol 31(3):176-187

20. Sutton D, Fuchs K, D'Alton M, Goffman D (2020) Universal Screening for SARS-CoV-2 in Women Admitted for Delivery. N Engl J Med

21. von Elm E, Altmann DG, Egger M, Pocock SJ, Gotzsche PC, Vandenbroucke JP, STROBE Initiative (2008) The Strengthening the Reporting of Observational Studies in Epidemiology (STROBE) Statement: Guidelines for Reporting Observational Studies. J Clin Epidemiol 61(4): 344-9

22. Wang C, Pan R, Wan X, Tan Y, Xu L, Ho CS, et al. (2020) Immediate Psychological Responses and Associated Factors during the Initial Stage of the 2019 Coronavirus Disease (COVID-19) Epidemic among the General Population in China. Int J Environ Res Public Health 17(5)

23. Williams VS, Morlock RJ, Feltner D (2010) Psychometric evaluation of a visual analog scale for the assessment of anxiety. Health Qual Life Outcomes 8:57

24. Yehuda R, Engel SM, Brand SR, Seckl J, Marcus SM, Berkowitz GS (2005) Transgenerational effects of posttraumatic stress disorder in babies of mothers exposed to the World Trade Center attacks during pregnancy. J Clin Endo Metab 90:4115-4118

\section{Tables}

Table 1: Demographics and pregnancy-related measures of 2740 pregnant women, regressed against change in Pregnancy-Related Anxiety Scale (PRAS) before and after COVID-19 


\begin{tabular}{|c|c|c|}
\hline Variable & $\begin{array}{l}\text { Overall } \\
\text { Mean (95\% } \\
\text { Cl) }\end{array}$ & $\beta(95 \% \mathrm{Cl}) ; \mathrm{P}$ value \\
\hline Maternal age (years) $(\mathrm{N}=2720)$ & $\begin{array}{l}32.7(32.5 \\
32.8)\end{array}$ & $\begin{array}{l}-0.10(-0.13,-0.07) \\
<0.001^{\star * \star}\end{array}$ \\
\hline Number of previous pregnancies & $1.7(1.6,1.7)$ & $-0.03(-0.11,0.06) ; 0.554$ \\
\hline \multirow[t]{2}{*}{ Number of prenatal care visits this pregnancy } & $6.5(6.3,6.6)$ & $0.03(-0.001,0.05) ; 0.065$ \\
\hline & $N(\%)$ & \\
\hline \multicolumn{3}{|l|}{ Region of residence $(\mathrm{N}=2695)$} \\
\hline Northeast & $536(19.9)$ & $0.06(-0.25,0.37) ; 0.707$ \\
\hline Midwest & $1159(43.0)$ & $-0.15(-.40,0.10) ; 0.240$ \\
\hline South & $619(22.9)$ & $0.21(-0.08,0.50) ; 0.159$ \\
\hline West & $381(14.1)$ & $-0.08(-0.44,0.27) ; 0.644$ \\
\hline \multicolumn{3}{|l|}{ Area of residence $(\mathrm{N}=2736)$} \\
\hline Urban & $725(26.5)$ & $0.12(-0.15,0.40) ; 0.385$ \\
\hline Peri-urban & $1559(56.9)$ & $-0.19(-0.44,0.05) ; 0.119$ \\
\hline Rural & $452(16.5)$ & $0.16(-0.17,0.49) ; 0.333$ \\
\hline \multicolumn{3}{|l|}{ Education $(\mathrm{N}=2740)$} \\
\hline High school graduate or less & $176(6.4)$ & $0.45(-0.07,0.97) ; 0.088$ \\
\hline College graduate or less & $1182(43.1)$ & REF \\
\hline Master's degree & $701(25.6)$ & $\begin{array}{l}-0.52(-0.83,-0.22) \\
0.001^{\star \star \star}\end{array}$ \\
\hline Doctoral/professional degree & $681(24.9)$ & $\begin{array}{l}-0.48(-0.78,-0.17) \\
0.002^{\star \star}\end{array}$ \\
\hline Caucasian Race (N=2721) & $2388(87.7)$ & $0.13(-0.25,0.50) ; 0.504$ \\
\hline Married $(\mathrm{N}=2740)$ & $2430(88.7)$ & $\begin{array}{l}-0.82(-1.20,-0.43) \\
<0.001^{\star \star \star}\end{array}$ \\
\hline In $3^{\text {rd }}$ trimester of pregnancy & $1128(41.2)$ & $0.30(0.05,0.55) ; 0.018^{*}$ \\
\hline First Pregnancy $(\mathrm{N}=2740)$ & $429(15.6)$ & $0.19(-0.15,0.53) ; 0.268$ \\
\hline Previous health conditions ${ }^{a}$ & $502(18.4)$ & $0.20(-0.11,0.52) ; 0.207$ \\
\hline Previous depression / anxiety & $982(35.9)$ & $0.39(0.13,0.64) ; 0.003^{\star *}$ \\
\hline
\end{tabular}




\begin{tabular}{|c|c|c|}
\hline Pregnancy health conditions ${ }^{b}$ & $526(19.2)$ & $0.29(-0.02,0.60) ; 0.071$ \\
\hline Pregnancy-diagnosed depression/anxiety & $154(5.6)$ & $\begin{array}{l}1.26(0.73,1.80) \\
<0.001^{\star \star \star}\end{array}$ \\
\hline Since COVID, stopped in-person PNC & $706(25.8)$ & $\begin{array}{l}0.54(0.26,0.82) \\
<0.001^{\star \star \star}\end{array}$ \\
\hline Since COVID, used video for PNC & $415(15.2)$ & $0.06(-0.29,0.40) ; 0.747$ \\
\hline Since COVID, used phone for PNC & $871(31.8)$ & $\begin{array}{l}0.46(0.20,0.72) \\
0.001^{\star \star \star}\end{array}$ \\
\hline \multicolumn{3}{|l|}{ Before COVID, planned location of birth: $(\mathrm{N}=2739)$} \\
\hline Hospital & $2641(96.4)$ & $0.97(0.31,1.63) ; 0.004^{\star \star}$ \\
\hline Birth center outside a hospital & $35(1.3)$ & $0.72(-0.37,1.81) 0.194$ \\
\hline Home & $39(1.4)$ & $\begin{array}{l}-2.50(-3.53,-1.47) \\
<0.001 * \star \star\end{array}$ \\
\hline Don't know & $24(0.9)$ & $-0.85(-2.16,0.46) ; 0.203$ \\
\hline \multicolumn{3}{|l|}{ Since COVID, planned location of birth: $(\mathrm{N}=2738)$} \\
\hline Hospital & $2400(87.7)$ & $\begin{array}{l}-0.99(-1.36,-0.62) \\
<0.001 \star \star \star\end{array}$ \\
\hline Birth center outside a hospital & $41(1.5)$ & $0.40(-0.60,1.41) ; 0.431$ \\
\hline Home & $74(2.7)$ & $\begin{array}{l}-1.24(-1.99,-0.49) \\
0.001^{\star \star \star}\end{array}$ \\
\hline Don't know & $223(8.1)$ & $\begin{array}{l}1.74(1.29,2.18) \\
<0.001 * * \star\end{array}$ \\
\hline $\begin{array}{l}\text { Birth location moved away from hospital (including } \\
\text { don't knows) }\end{array}$ & $241(8.8)$ & $\begin{array}{l}1.76(1.32,2.20) \\
p<0.001^{\star \star \star}\end{array}$ \\
\hline
\end{tabular}

${ }^{a}$ Diagnosed prior to pregnancy with hypertension, heart disease, asthma, diabetes, cancer, autoimmune diseases, or HIV/AIDS

${ }^{\mathrm{b}}$ During pregnancy, diagnosed with hypertension, preeclampsia, gestational diabetes, anemia, placenta previa

*p0.05; **p0.01; ***p0.001

Table 2: Anxiety measures and psychosocial risk ractors related to COVID-19, regressed againsted change in self-reported Pregnancy-Related Anxiety Scale (PRAS) before and after COVID-19 


\begin{tabular}{|c|c|c|}
\hline Variable & Overall $(\mathrm{N}=\mathbf{2 7 4 0})$ & $\beta(95 \% \mathrm{Cl}) ; \mathrm{P}$ value \\
\hline & Mean $(95 \% \mathrm{Cl})$ & \\
\hline Anxiety about being pregnant during COVID ${ }^{a}$ & $6.5(6.4,6.6)$ & $0.61(0.56,0.66) ;<0.001 * \star *$ \\
\hline Anxiety about giving birth during COVID ${ }^{a}$ & $7.6(7.5,7.6)$ & $0.54(0.49,0.59) ;<0.001^{\star \star \star}$ \\
\hline Pre-COVID PRAS score & $20.6(20.5,20.7)$ & - \\
\hline Post-COVID PRAS score & $23.9(23.8,24.0)$ & - \\
\hline \multicolumn{3}{|l|}{ Since COVID, increased stress about... } \\
\hline Food running out /availability & $1622(59.2)$ & $1.24(0.99,1.49) ;<0.001^{\star \star \star}$ \\
\hline Losing a job / loss of income & $1745(63.7)$ & $1.16(0.91,1.41) ;<0.001^{\star \star \star}$ \\
\hline Loss of childcare & $1543(56.3)$ & $0.42(0.18,0.67) ; 0.001^{\star \star \star}$ \\
\hline Tension / conflict in house & $1028(37.5)$ & $0.78(0.53,1.03) ;<0.001^{\star * *}$ \\
\hline Getting infected & $2556(93.3)$ & $1.82(1.34,2.31) ;<0.001 * \star \star$ \\
\hline \multicolumn{3}{|l|}{ Risk Factors for COVID } \\
\hline Self / family healthcare worker & $1133(41.4)$ & $-0.07(-0.32,0.18) ; 0.566$ \\
\hline Self / family essential worker & $1246(45.5)$ & $0.52(0.27,0.76) ;<0.001 \star \star \star$ \\
\hline Live with 'shelter in place' orders & $2117(77.3)$ & $-0.34(-0.63,-0.05) ; 0.021 \star \star$ \\
\hline Practicing social distancing & $2395(87.4)$ & $-0.62(-0.99,-0.25) ; 0.001 \star \star \star$ \\
\hline Live in high COVID community & 1149 (41.9) & $0.41(0.16,0.65) ; 0.001^{\star \star \star}$ \\
\hline
\end{tabular}

a 1-10 Visual Analog Scale with 1 being not at all and 10 being extremely anxious *p $0.05 ; * \star p 0.01 ; * \star \star p 0.001$

Table 3: Multivariate linear regression reflecting factors associated with change in pre- to post-COVID Pregancy-Related Anxiety Scale (PRAS) scores 
Variable

Demographic and pregnancy-related factors

Maternal age (years)

$-0.08$

$-0.10,-0.05$

$<0.001^{\star \star \star}$

Education (ref: college graduate/less)

High school graduate or less

$-1.17 \quad-2.25,-0.09$

$0.034^{\star}$

Master's degree

Doctoral / professional degree

In 3rd trimester of pregnancy

Pregnancy-diagnosed depression / anxiety

Since COVID, stopped in-person PNC

Since COVID, changed birth plan away from delivery in a hospital

Anxiety and psychosocial risk factors

Since COVID, increased stress about...

Food running out / availability

0.77

0.75

0.12

$-0.22$

$-0.69,0.30$;

0.372

0.50

$-0.37,0.61$

0.637

$0.26,0.74$ $<0.001^{\star \star \star}$

$0.24,1.26$

$0.004^{\star *}$

0.42

$0.15,0.69$

$0.002^{\star *}$

1.26

$0.84,1.68$ $<0.001^{\star \star *}$

Losing a job / loss of income

$0.47 \quad-0.01,0.94$

0.053

Tension / conflict in house

0.49

$0.25,0.73$ $<0.001 * \star \star$

Getting infected

1.23

$0.76,1.71$ $<0.001^{\star \star \star}$

Risk Factors for COVID

Self / family member essential worker

0.42

$0.18,0.66$

$0.001^{* \star}$

Practicing social distancing

$-0.86$

$-1.23,-0.49$ $<0.001^{\star \star \star}$

Live in high COVID community

0.42

$0.17,0.67$

$0.001^{\star *}$

Interaction between anxious about loss of income or job and high school

$1.320 .08,2.57$ 
${ }^{*} \mathrm{p}<=0.05,{ }^{* *} \mathrm{p}<=.01 ;{ }^{* *} \mathrm{p}<=001$

\section{Supplementary Files}

This is a list of supplementary files associated with this preprint. Click to download.

- Appendix1.docx 